\title{
Co and Phthalocyanine Overlayers on the Quantum-Well System Co(001)/Cu: Spin-Polarized Electron Reflection Experiments
}

\author{
Etienne Urbain1, Guillaume Garreau², Patrick Wetzel'2, Samy Boukari1', Eric Beaurepaire1, \\ Martin Bowen', Wolfgang Weber ${ }^{1}$
}

${ }^{1}$ Université de Strasbourg, CNRS, Institut de Physique et Chimie des Matériaux de Strasbourg, Strasbourg, France

${ }^{2}$ Université de Haute-Alsace, CNRS, Institut de Science des Matériaux de Mulhouse, Mulhouse, France

Email: wolfgang.weber@ipcms.unistra.fr

How to cite this paper: Urbain, E., Garreau, G., Wetzel, P., Boukari, S., Beaurepaire, E., Bowen, M. and Weber, W. (2018) Co and Phthalocyanine Overlayers on the Quantum-Well System $\mathrm{Co}(001) / \mathrm{Cu}$ : Spin-Polarized Electron Reflection Experiments. Journal of Modern Physics, 9, 976-984.

https://doi.org/10.4236/jmp.2018.95060

Received: March 10, 2018

Accepted: April 13, 2018

Published: April 16, 2018

Copyright (c) 2018 by authors and Scientific Research Publishing Inc. This work is licensed under the Creative Commons Attribution International License (CC BY 4.0).

http://creativecommons.org/licenses/by/4.0/

\begin{abstract}
The influence of a Co or phthalocyanine $(\mathrm{Pc})$ molecular overlayer on the properties of quantum-well resonances (QWR) in $\mathrm{Cu}$ layers atop $\mathrm{Co}(001)$ is studied by means of spin-polarized electron reflection. For Co atoms and Pc molecules, an energy shift of the QWR-induced signal is observed with increasing coverage and is attributed to a variation of the electron reflection phase at the $\mathrm{Cu} / \mathrm{Co}$ and $\mathrm{Cu} / \mathrm{Pc}$ interface. For Co we find a linear energy shift in the $\mathrm{Cu}$ QWR energy position with increasing coverage down to the sub-monolayer regime. This shows that the phase accumulation model remains accurate within the sub-monolayer regime of a discontinuous interface. An opposite sign in the energy shift between Co and Pc overlayers could reflect an opposite impact on the $\mathrm{Cu}$ surface work function of overlayer adsorption.
\end{abstract}

\section{Keywords}

Quantum-Well Resonances, Spin-Polarized Electron Reflection

\section{Introduction}

Quantum confinement in thin metallic films gives rise to discrete quantum-well states (see Ref. [1] and references therein) that are known to lead, as the film thickness is varied, to variations in different physical quantities such as surface energy [2], thermal stability [3], work function [4], electrical conductivity [5] and surface adsorption [6] [7]. This confinement can become spin-dependent if ferromagnetic layers are considered. This accounts for the oscillatory behavior of the indirect exchange coupling between two ferromagnetic layers separated by a 
non-magnetic spacer layer [8], the magneto-optical response [9], the induced magnetic moment [10], the magnetic anisotropy [11], and the Curie temperature [12].

While there is a lot of literature concerning quantum confinement in systems such as $\mathrm{Fe}(001) / \mathrm{Ag}$ or $\mathrm{Co}(001) / \mathrm{Cu}$ (for instance Refs. [1] and [13]), only little work exists on how quantum well states (QWS; below the vacuum energy) or resonances (above the vacuum energy) are influenced when such a system is covered. Scanning tunneling spectroscopy measurements on QWS in $\mathrm{Cu}$ and $\mathrm{Pb}$ studied the behavior of the Kondo resonance of Co for Co overlayers [14] [15] and of $\mathrm{Mn}$ for a molecular overlayer coverage of Mn-phthalocyanine (MnPc) [16]. As the Kondo resonance can only be seen for isolated atoms or molecules only very small coverage of about 0.01 monolayer (ML) could be studied. Interestingly, quite different results were obtained for Co coverage on $\mathrm{Cu}$ [14] and on $\mathrm{Pb}$ [15]. While the Kondo resonance of Co atoms on $\mathrm{Cu}$ shows QWS-induced oscillatory modulations of its line width, Co atoms adsorbed atop $\mathrm{Pb}$ destroy the QWS within the $\mathrm{Pb}$ film. On the other hand, the QWS in the Pb layer are not destroyed by adsorption of MnPc molecules which is proven by the presence of strong line width modulations of the Kondo resonance of Mn [16]. The effect on QWS in Ag(111) layers by a very similar molecule, namely CuPc, has been studied by photoemission spectroscopy [17]. Interestingly, neither the intensities of the QWS (after consideration of the signal attenuation due to the $\mathrm{CuPc}$ layer) nor their energy positions change with increasing $\mathrm{CuPc}$ coverage (up to $1 \mathrm{ML})$.

The goal of this work is to shed more light on the question of how the presence of an additional interface affects the quantum confinement, in particular as the above cited results are somewhat contradictory. In the following we examine the behavior of QWR by spin-polarized reflection of electrons in $\operatorname{Co}(001) / \mathrm{Cu}$ layers when covered by metallic Co or by semiconducting Pc molecules.

Information on QWS are mostly obtained via photoemission experiments [13]. One of the major results of such experiments is that the appearance of QWS as a function of film thickness is explained very well by the so-called phase accumulation model [18]. Consequently, if the electron system is confined to a thin film of thickness $d$ deposited onto a substrate, constructive interference, i.e. maximum in intensity, requires the wave vector component $k_{\perp}$ perpendicular to the surface of the electrons in the thin film to fulfill the standard quantization condition:

$$
2 k_{\perp} d+\theta=2 \pi n
$$

with $\theta$ a phase shift due to the reflections of the electrons at the film/vacuum and the substrate/film interface and $n$ an integer. As a consequence, the difference between two neighboring $k_{\perp}$-values fulfilling the above interference condition decreases with increasing film thickness. Thus, the number of QWS increases with increasing film thickness. 


\section{Experiment}

The experimental set-up is shown schematically in Figure 1. A spin-polarized electron-source based on a GaAs photocathode produces a spin-polarized electron beam with $25 \%$ spin polarization [19]. By switching from right- to left-circularly polarized light, the direction of the initial spin polarization of the photoexcited electrons can be inverted. The electron beam impinges onto the sample at an angle of $45^{\circ}$ with respect to the surface normal [001] of the $\mathrm{Cu}(001)$ single crystal. The surface projection of the incident electron wave vector is along the [110]-direction of the $\mathrm{Cu}$ substrate. The spin polarization vector $\boldsymbol{P}_{0}$ of the incident electrons is oriented either parallel or antiparallel to the magnetization vector $\boldsymbol{M}$ of the ferromagnetic material. The specularly reflected electrons are energy analyzed by a retarding grid analyzer that has an energy resolution of $0.3 \mathrm{eV}$ FWHM. The broad distribution of inelastically scattered electrons is suppressed by applying a retarding field to the retardation grid. Subsequently, the intensity of the specularly reflected electron beam is measured. To get rid of any experimental asymmetry, the direction in space as well as the relative alignment of $\boldsymbol{P}_{0}$ and $\boldsymbol{M}$ is interchanged.

Due to exchange interactions at the surface of the ferromagnetic sample, which are essentially an outcome of the Pauli principle, the reflection properties of the electron beam depend on the relative orientation of $\boldsymbol{P}_{0}$ and $\boldsymbol{M}$. In fact, the reflectivities for electrons with $P_{0}$ parallel to $M, R_{\uparrow \uparrow}$, and antiparallel to $M$, $R_{\uparrow \downarrow}$, are in general different, such that one obtains a non-vanishing exchange asymmetry:

$$
A_{e x}=\frac{1}{P_{0}} \frac{R_{\uparrow \uparrow}-R_{\uparrow \downarrow}}{R_{\uparrow \uparrow}+R_{\uparrow \downarrow}}
$$

which is normalized by the polarization value $P_{0}$ of the incident electron beam.

In a first step a Co film of $10 \mathrm{ML}$ thickness was deposited onto a single crystalline $\mathrm{Cu}(001)$ substrate at room temperature from a Co rod heated by electron beam bombardment. Prior to deposition, the $\mathrm{Cu}$ substrate was cleaned by several

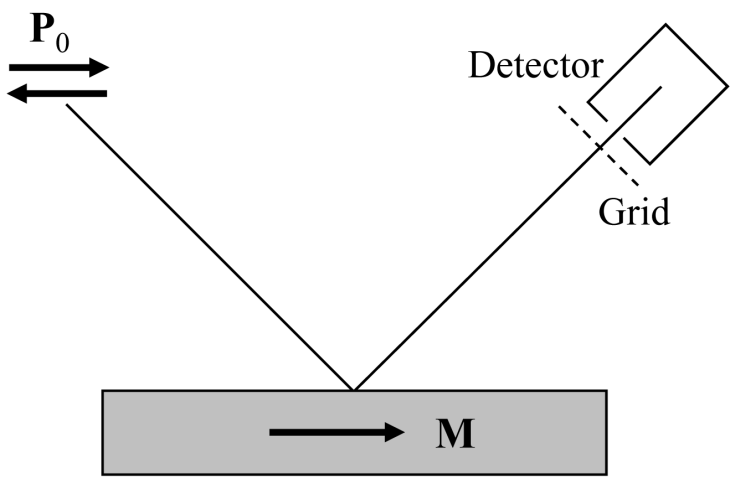

Figure 1. The principle of the experiment. It consists of a spin-polarized electron source creating electrons with spin polarization $\boldsymbol{P}_{0}$, a sample with magnetization $\boldsymbol{M}$, a retardation grid for energy analysis, and an electron detection system. 
cycles of Ar-ion sputtering and annealing at $800 \mathrm{~K}$. Co on $\mathrm{Cu}(001)$ exhibits an easy axis of magnetization in the film plane along the [110] direction. The Co films are magnetized remanently by applying magnetic field pulses along this direction. We note that the system $\mathrm{Cu}(001) / \mathrm{Co}$ has extensively been investigated in the past (see for instance Refs. [20] [21] [22] [23]). In a second step Cu films of different thicknesses $(3,6$, and $10 \mathrm{ML})$ are deposited onto the Co film at room temperature. This overlayer system, namely $\mathrm{Co}(001) / \mathrm{Cu}$, has as well extensively been studied in the past and shows the existence of QWS and QWR [24] [25]. Both $\mathrm{Co}$ and $\mathrm{Cu}$ thicknesses could be determined within an accuracy of about $\pm 10 \%$. The evaporation rate was controlled by a quartz microbalance. In a third step either $\mathrm{Co}$ or $\mathrm{H}_{2}$-phthalocyanine $\left(\mathrm{H}_{2} \mathrm{Pc}\right)$ molecules are deposited at room temperature successively onto the $\mathrm{Co} / \mathrm{Cu}$ stack during the electron reflection experiments. For detailed information about the growth of Pc molecules on $\mathrm{Cu}(001)$ in the monolayer range see Ref. [26]. The thicknesses of the molecular layers are determined by Auger electron spectroscopy [27] and by the study of the work function change with increasing Pc thickness [27]. We emphasize that our X-ray photoemission spectroscopy measurements on $\mathrm{H}_{2} \mathrm{Pc}$ molecules deposited onto $\mathrm{Cu}(001)$ at room temperature do not indicate any $\mathrm{Cu}$-metalation of the $\mathrm{H}_{2} \mathrm{Pc}$ molecules (i.e. replacement of the two central protons by $\mathrm{Cu}^{2+}$; data not shown).

\section{Results and Discussion}

It has already been shown by Egger et al. [28] that the very pronounced structures of $A_{e x}$ as a function of $\mathrm{Cu}$ thickness in the system $\mathrm{Co}(001) / \mathrm{Cu}$ are directly related to the presence of QWR in the $\mathrm{Cu}$ film. In a later work [25] the $\mathrm{Cu}$-thickness dependent behavior of the spin motion angle $\phi$, which is directly related to the quantity $A_{e x}$ [29], has been studied. Most importantly, the energy positions of the extrema in $\phi$ and thus in $A_{e x}$ shift with varying $\mathrm{Cu}$ film thickness and are in good agreement with those of the extrema in reflectivity. This shows that the existence of QWR is at the origin not only of the reflectivity oscillations but also of the oscillations in $A_{e x}$ Moreover, the extrema in $A_{e x}$ usually are much more pronounced than those of the reflectivity [25] [30] such that $A_{e x}$ is an ideal quantity to study the behavior of QWR.

We present in Figure 2 the dependence of $A_{e x}$ on the kinetic electron energy (i.e. the incident energy with respect to the vacuum level) as the thickness of the Co and Pc overlayer is increased. In both cases, we concentrate on the energy variation of the $A_{e x}$ minimum between 6 and $7 \mathrm{eV}$ kinetic energy. While an energy shift in the $A_{e x}$ minimum to lower kinetic energies is observed with increasing Co coverage, the energy shift is positive for Pc coverage. We emphasize that the observed energy shifts of the QWR-induced structure is related to a phase shift. However, we cannot extract phase shifts directly from the measured energy shifts as the phase change $\Delta \theta$ is proportional to the change $\Delta k_{\perp}$ of the electron wave vector, $\Delta \theta=-2 d \cdot \Delta k_{\perp}$, and not of the energy difference (see 

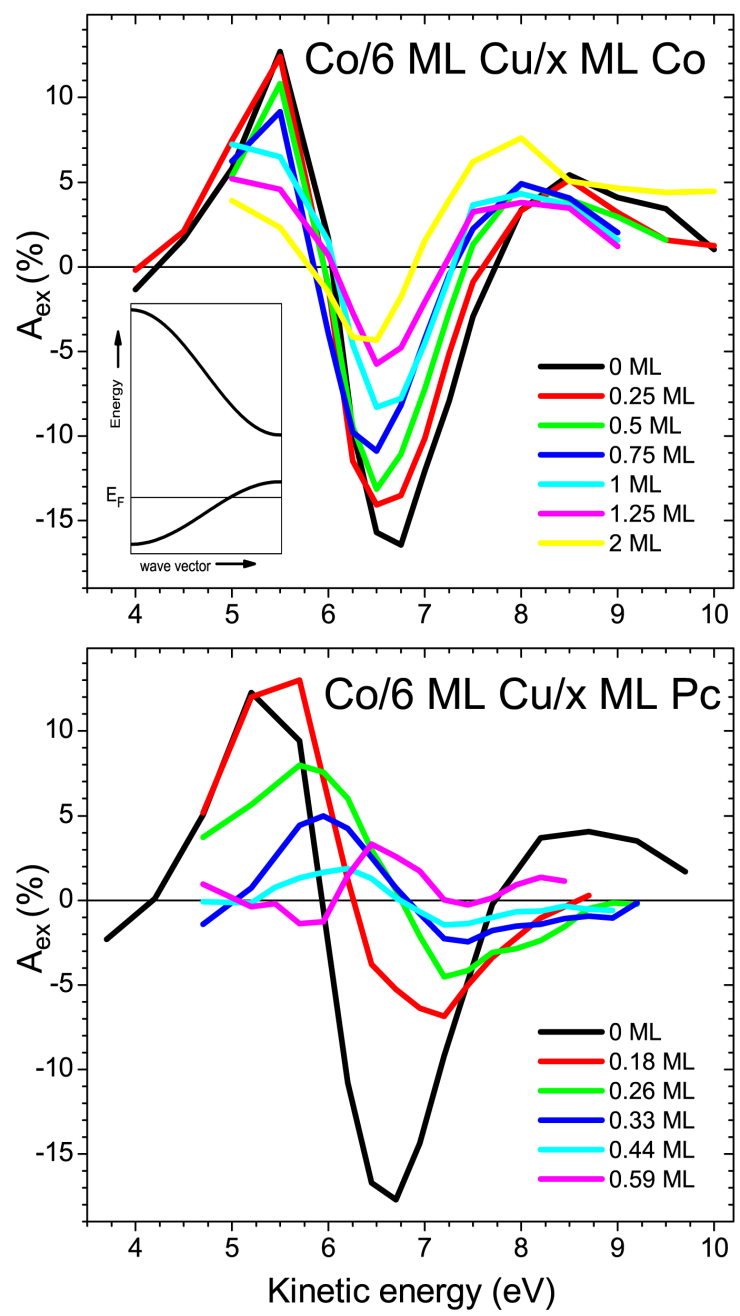

Figure 2. $A_{e x}$ vs. kinetic electron energy for different Co (top) and Pc coverages (bottom) on $6 \mathrm{ML} \mathrm{Cu}$ on $\mathrm{Co}(001)$. The inset shows a schematic electronic band structure with two bands and a band gap in-between (see text).

Equation (1)). In order to obtain the corresponding $k_{\perp}$ values one needs thus the unoccupied band structure of $\mathrm{Cu}$ above the vacuum level. This, however, is not available for our particular measuring geometry in which the incident electron wave vector is not normal to the sample surface (see section "Experiment") so that we have to refrain from a calculation of the phase shifts and show only the energy shifts.

We plot in Figure 3 the energy shift of the QWR-induced $A_{e x}$ minimum for both $\mathrm{Co}$ and $\mathrm{Pc}$ coverage on $\mathrm{Co}(001) / \mathrm{Cu}$ with varying $\mathrm{Cu}$ thickness. We first discuss the case of the Co overlayer. This energy shift is linear within the Co thickness range studied, with a slope that decreases when going from 3 to $10 \mathrm{ML}$ $\mathrm{Cu}$ thickness. This is understandable since, with increasing $\mathrm{Cu}$ thickness, the QWS features become denser in energy [1], such that a given phase shift corresponds to a smaller energy shift. The observation that the energy position of the QWR shifts to lower kinetic energy is consistent with $a b$ initio calculations of a 


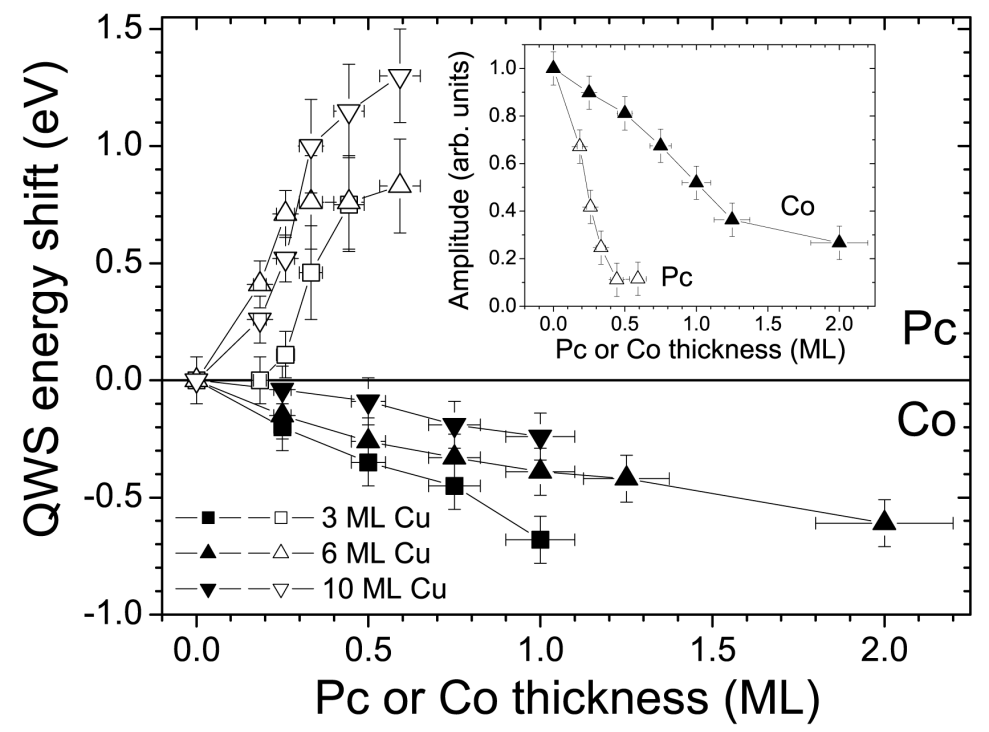

Figure 3. The energy shift of the QWR-induced $A_{e x}$ structure for Co coverage (full symbols) and Pc coverage (open symbols) on $\mathrm{Co}(001) / \mathrm{Cu}$ with varying $\mathrm{Cu}$ thickness. The inset shows the amplitude of the $A_{e x}$ structure as a function of both Co and Pc coverage.

Co overlayer on top of the $\mathrm{Co}(001) / \mathrm{Cu}$ quantum-well system [31]. In fact, these calculations of the density-of-states below $E_{F}$ show that with increasing Co coverage the QWS disperse smoothly upwards in energy. This is due to the fact that the wave vector of the QWS which lie on the lower band (see the schematic band structure as inset of Figure 2 (top)) shifts to higher values such that the QWS come closer to the boundary of the $\mathrm{Cu}$ Brillouin zone. In the case of QWR which lie above the band gap (see inset of Figure 2 (top)) this wave-vector shift closer to the Brillouin zone boundary leads thus to a downward dispersion in energy of the QWR with increasing Co thickness as observed.

Our result is particularly interesting in view of recent calculations concerning the effect of Co ad-atoms on the quantum confinement in the $\mathrm{Co}(001) / \mathrm{Cu}$ system [32]. These calculations show that the density-of-states of the $\mathrm{Cu}$ surface is drastically modified by a sub-ML fraction of Co ad-atoms, such that an analysis within the phase accumulation model proves to be inadequate. This failure of the phase accumulation model suggests that the QWS are substantially perturbed by the presence of Co ad-atoms, which, in contrast to a complete monolayer, do not constitute a smooth interface. However, within our study we observe a linear increase of the energy shift between 0 and 2 ML Co coverage. There is thus no experimental indication that the behavior of an incomplete Co layer might be very different from that of a complete Co layer. The lateral inhomogeneity of the Co film for sub-ML coverage does not seem to be important at least for the energy shift and thus the phase shift. Moreover, a strongly perturbed QWR would result in a strongly modified amplitude of the $A_{e x}$ signal. However, this is not observed in our measurements. In fact, the inset in Figure 3 shows only a monotonous decrease of the $A_{e x}$-amplitude whose value is mainly determined by the limited 
mean free path of the electrons.

The behavior of the energy shift as a function of Pc coverage is quite different from that of Co coverage. The most important difference concerns the amplitude of the $A_{e x}$ signal as the latter is rapidly decreasing with increasing Pc coverage and almost disappears for coverages well below $1 \mathrm{ML}$ (see inset in Figure 3). We emphasize that a similar behavior, which we called breakdown phenomenon and which results in a spin-independence of the electron reflection (i.e. $A_{e x}$ becomes zero), has been found recently by some of us for many metal-organic interfaces [27] [33]. It is important to note that this breakdown phenomenon is only observed in experiments in which the reflection of electrons is studied and is not yet understood. Thus, a rapid decrease of the $A_{e x}$ signal with increasing Pc coverage is indeed expected. Within the Pc coverage range for which the $A_{e x}$ signal can be studied as a function of energy, we observe an energy shift that is much stronger than that of Co. Moreover, it does not exhibit a linear behavior as for Co and seems to saturate. This behavior of the energy shift thus resembles that of the breakdown phenomenon and is as such without explanation at the moment.

The other significant difference between the case of Co and Pc overlayers is the opposite sign in the QWR energy shifts. However, this does not seem to us very surprising. As the electronic density-of-states of Co and Pc are completely different one can expect as well quite different electron reflection properties at the interfaces $\mathrm{Cu} / \mathrm{Co}$ and $\mathrm{Cu} / \mathrm{Pc}$, including opposite sign of the phase shifts. Moreover, the change of the work function at the overlayer surface might also have a certain influence on the reflection properties of the standing electron waves [1], in particular if the overlayer is extremely thin, as it is the case in our measurements, such that the overlayer/vacuum interface can have an important influence on the $\mathrm{Cu}$ /overlayer interface. Since the work function differences $\Delta \phi$ in the two cases are in particular of opposite sign $(\mathrm{Cu} / \mathrm{Co}: \Delta \phi=+0.3 \mathrm{eV}, \mathrm{Cu} / 1$ ML Pc: $\Delta \phi=-0.8 \mathrm{eV}$ [34]) they could be responsible for the different sign in phase shift.

\section{Conclusion}

In conclusion, the spin-dependent electron reflection at the $\mathrm{Cu} / \mathrm{Co}$ or $\mathrm{Cu} / \mathrm{Pc}$ top interface within the $\mathrm{Co}(001) / \mathrm{Cu}$ quantum-well system has been studied. A clear energy shift of the QWR-induced signal was observed both for Co atoms and Pc molecules with increasing overlayer coverage. The linearity of this energy shift for increasing Co coverage, both below and above the monolayer, suggests that the phase accumulation model remains valid below the monolayer regime for the quantum well considered. A comparison with the literature suggests that the inferred variation of the electron reflection phase at the $\mathrm{Cu} / \mathrm{Co}$ and $\mathrm{Cu} /$ molecule interface appears to depend on the exact quantum well resonance considered. The opposite sign of the energy shift for Co and Pc overlayers could reflect the opposite impact on the $\mathrm{Cu}$ surface work function of these overlayers. This work 
establishes guidelines to better understand the physics of spin-polarized quantum confinement using both metallic and molecular layers.

\section{References}

[1] Milun, M., Pervan, P. and Woodruff, D.P. (2002) Reports on Progress in Physics, 65, 99-141. https://doi.org/10.1088/0034-4885/65/2/201

[2] Wei, C.M. and Chou, M.Y. (2002) Physical Review B, 66, Article ID: 233408.

[3] Upton, M.H., Wei, C.M., Chou, M.Y., Miller, T. and Chiang, T.-C. (2004) Physical Review Letters, 93, Article ID: 026802. https://doi.org/10.1103/PhysRevLett.93.026802

[4] Kim, J., Qin, S., Yao, W., Niu, Q., Chou, M.Y. and Shih, C.-K. (2009) Proceedings of the National Academy of Sciences of the United States of America, 107, 12761. https://doi.org/10.1073/pnas.0915171107

[5] Miyata, N., Horikoshi, K., Hirahara, T., Hasegawa, S., Wei, C.M. and Matsuda, I. (2008) Physical Review B, 78, Article ID: 245405. https://doi.org/10.1103/PhysRevB.78.245405

[6] Aballe, L., Barinov, A., Locatelli, A., Heun, S. and Kiskinova, M. (2004) Physical Review Letters, 93, Article ID: 196103. https://doi.org/10.1103/PhysRevLett.93.196103

[7] Ma, X., Jiang, P., Qi, Y., Jia, J., Yang, Y., Duan, W., Li, W.-X., Bao, X., Zhang, S.B. and Xue, K.-Q. (2007) Proceedings of the National Academy of Sciences of the United States of America, 104, 9204-9208. https://doi.org/10.1073/pnas.0611024104

[8] Parkin, S.S.P., More, N. and Roche, K.P. (1990) Physical Review Letters, 64, 2304-2307. https://doi.org/10.1103/PhysRevLett.64.2304

[9] Bruno, P., Suzuki, Y. and Chappert, C. (1996) Physical Review B, 53, 9214. https://doi.org/10.1103/PhysRevB.53.9214

[10] Back, C.H., Weber, W., Bischof, A., Pescia, D. and Allenspach, R. (1995) Physical Review B, 52, R13114. https://doi.org/10.1103/PhysRevB.52.R13114

[11] Weber, W., Bischof, A., Allenspach, R., Würsch, C., Back, C.H. and Pescia, D. (1996) Physical Review Letters, 76, 3424-3427. https://doi.org/10.1103/PhysRevLett.76.3424

[12] Ney, A., Wilhelm, F., Farle, M., Poulopoulos, P., Srivastava, P. and Baberschke, K. (1999) Physical Review B, 59, R3938. https://doi.org/10.1103/PhysRevB.59.R3938

[13] Chiang, T.-C. (2000) Surface Science Reports, 39, 181-235. https://doi.org/10.1016/S0167-5729(00)00006-6

[14] Uchihashi, T., Zhang, J., Kröger, J. and Berndt, R. (2008) Physical Review B, 78, Article ID: 033402. https://doi.org/10.1103/PhysRevB.78.033402

[15] Zhang, X., Zhao, A., Wang, K. and Xiao, X. (2008) Physical Review B, 78, Article ID: 035431 .

[16] Fu, Y.-S., Ji, S.-H., Chen, X., Ma, X.-C., Wu, R., Wang, C.-C., Duan, W.-H., Qiu, X.-H., Sun, B., Zhang, P., Jia, J.-F. and Xue, Q.-K. (2007) Physical Review Letters, 99, Article ID: 256601. https://doi.org/10.1103/PhysRevLett.99.256601

[17] Lin, M.-K., Nakayama, Y., Chen, C.-H., Wang, C.-Y., Jeng, H.-T., Pi, T.-W., Ishii, H. and Tang, S.-J. (2013) Nature Communications, 4, Article No. 2925. https://doi.org/10.1038/ncomms3925

[18] Mueller, M.A., Miller, T. and Chiang, T.-C. (1990) Physical Review B, 41, 5214-5220. https://doi.org/10.1103/PhysRevB.41.5214 
[19] Pierce, D.T., Celotta, R.J., Wang, G.C., Unertl, W.N., Galejs, A., Kuyatt, C.E. and Mielczarek, S.R. (1980) Review of Scientific Instruments, 51, 478-499. https://doi.org/10.1063/1.1136250

[20] Clarke, A., Jennings, G., Willis, R.F., Rous, P.J. and Pendry, J.B. (1987) Surface Science, 187, 327-338. https://doi.org/10.1016/S0039-6028(87)80060-2

[21] Krams, P., Lauks, F., Stamps, R.L., Hillebrands, B. and Güntherodt, G. (1992) Physical Review Letters, 69, 3674-3677. https://doi.org/10.1103/PhysRevLett.69.3674

[22] Heckmann, O., Magnan, H., le Fevre, P., Chandesris, D. and Rehr, J.J. (1994) Surface Science, 312, 62-72. https://doi.org/10.1016/0039-6028(94)90803-6

[23] Ramsperger, U., Vaterlaus, A., Pfäffli, P., Maier, U. and Pescia, D. (1996) Physical Review B, 53, 8001-8006. https://doi.org/10.1103/PhysRevB.53.8001

[24] Ortega, J.E., Himpsel, F.J., Mankey, G.J. and Willis, R.F. (1993) Physical Review B, 47, 1540-1552. https://doi.org/10.1103/PhysRevB.47.1540

[25] Joly, L., Tati-Bismaths, L. and Weber, W. (2006) Physical Review Letters, 97, Article ID: 187404. https://doi.org/10.1103/PhysRevLett.97.187404

[26] Guo, Q., Qin, Z., Zang, K., Liu, C., Yu, Y. and Cao, G. (2010) Langmuir, 26, 11804-11808. https://doi.org/10.1021/la1019907

[27] Djeghloul, F., Dey, P., Hallal, A., Urbain, E., Mahiddine, S., Gruber, M., Spor, D., Alouani, M., Bulou, H., Scheurer, F. and Weber, W. (2014) Physical Review B, 89, Article ID: 134411. https://doi.org/10.1103/PhysRevB.89.134411

[28] Egger, S., Back, C.H., Krewer, J. and Pescia, D. (1999) Physical Review Letters, 83, 2833-2836. https://doi.org/10.1103/PhysRevLett.83.2833

[29] Dey, P. and Weber, W. (2011) Journal of Physics: Condensed Matte, 23, Article ID: 473201. https://doi.org/10.1088/0953-8984/23/47/473201

[30] Joly, L., Tati-Bismaths, L., Scheurer, F. and Weber, W. (2007) Physical Review B, 76, Article ID: 104415. https://doi.org/10.1103/PhysRevB.76.104415

[31] van Gelderen, P., Crampin, S. and Inglesfield, J.E. (1996) Physical Review B, 53, 9115. https://doi.org/10.1103/PhysRevB.53.9115

[32] Schwingenschlögl, U., Uchihashi, T., Di Paola, C. and Berndt, R. (2010) Physical Review B, 82, Article ID: 033406. https://doi.org/10.1103/PhysRevB.82.033406

[33] Ochapski, M., Urbain, E., Djeghloul, F., Speisser, V., Majjad, H., Spor, D., Vu, A.D., Coraux, J., Rougemaille, N., Chen, G., Schmid, A.K., Suzuki, M., Yasue, T., Koshikawa, T., Bulou, H. and Weber, W. (2016) Physical Review B, 93, Article ID: 174411. https://doi.org/10.1103/PhysRevB.93.174411

[34] Javaid, S., Lebégue, S., Detlefs, B., Ibrahim, F., Djeghloul, F., Bowen, M., Boukari, S., Miyamachi, T., Arabski, J., Spor, D., Zegenhagen, J., Wulfhekel, W., Weber, W., Beaurepaire, E. and Alouani, M. (2013) Physical Review B, 87, Article ID: 155418. https://doi.org/10.1103/PhysRevB.87.155418 